\title{
Diseño, construcción y control de un convertidor de potencia de cinco niveles
}

\author{
Antonio Ventosa-Cutillas \\ Escuela Técnica Superior de Ingeniería, Universidad de Sevilla, aventosa@us.es \\ Marta Gómez-Correa \\ Escuela Técnica Superior de Ingeniería, Universidad de Sevilla, mgomez42@us.es \\ Francisco Gordillo \\ Escuela Técnica Superior de Ingeniería, Universidad de Sevilla, gordillo@us.es \\ Federico Cuesta \\ Escuela Técnica Superior de Ingeniería, Universidad de Sevilla, fede@cartuja.us.es
}

\begin{abstract}
Resumen
En el trabajo que aquí se presenta se va a exponer el diseño, construcción y control de un convertidor de cinco niveles de diodos fijos funcionando en modo rectificador. Dada la complejidad a la hora de conseguir buenos resultados de control, este tipo de convertidores se encuentran principalmente destinados a la investigación, razón por la cual supone un reto desde el punto de vista del control de la tensión en el bus de continua y del balance o equilibrio de los condensadores.

Palabras clave: Convertidor de potencia de diodos fijos (DCC), Rectificador de cinco niveles, Diseño y montaje de un rectificador, Balance de tensiones.
\end{abstract}

\section{INTRODUCCIÓN}

En la última década, los convertidores multinivel han experimentado una creciente implantación en la industria y un notable aumento como objeto de investigación [4], [2].

Así como los convertidores de tres niveles son bastante utilizados en diversas aplicaciones industriales, los convertidores de mayor número de niveles están más destinados a tareas de investigación debido a su mayor coste y a la complejidad que presenta el desarrollo de leyes de control. Concretamente, presenta especial dificultad mantener el balance de tensiones en los condensadores del bus de continua cuando se aplican técnicas convencionales de modulación [5].

Sin embargo, los convertidores multinivel presentan una serie de ventajas [4]:
- Pueden generar voltajes de salida con muy baja distorsión y bajo $d v / d t$.

- Distorsionan muy poco la corriente a la entrada.

- Generan bajas tensiones de modo común, tensión entre el punto medio de los condensadores y tierra, reduciendo el estrés en los cojinetes de los motores.

- Son capaces de funcionar a frecuencias de conmutación bajas, reduciendo de esta forma las pérdidas por conmutación y mejorando el rendimiento.

Un ejemplo de aplicaciones en las que se utilizan estos convertidores son para uso en conversión y generación de energía, fabricación, transporte o transmisión de potencia [4], [7], [3].

Existen diversos tipos de convertidores multinivel, siendo los más conocidos los convertidores de diodos fijos (DCC), capacidades flotantes (FC) y puente en $\mathrm{H}$ en cascada (CHB) [3], [1].

El tipo de convertidor a utilizar dependerá de la aplicación para la que se va a destinar, teniendo en cuenta las características y limitaciones de cada tipo de convertidor, tales como potencia y tensión de uso, rendimiento, fiabilidad, coste y otros aspectos técnicos.

En el trabajo que aquí se presenta, se ha diseñado un convertidor de diodos fijos de cinco niveles, cuyo circuito se puede observar en la figura 1. Para ello, se ha tenido en cuenta que la tensión de alimentación será una red trifásica y que la salida será una tensión continua de unos 800 voltios, obteniéndose así un equipo de unos $11 \mathrm{KW}$ de potencia. Para el diseño de este convertidor se ha utilizado un equipo de control en tiempo real llamado Speedgoat, cuyas características se explicarán más adelante. 


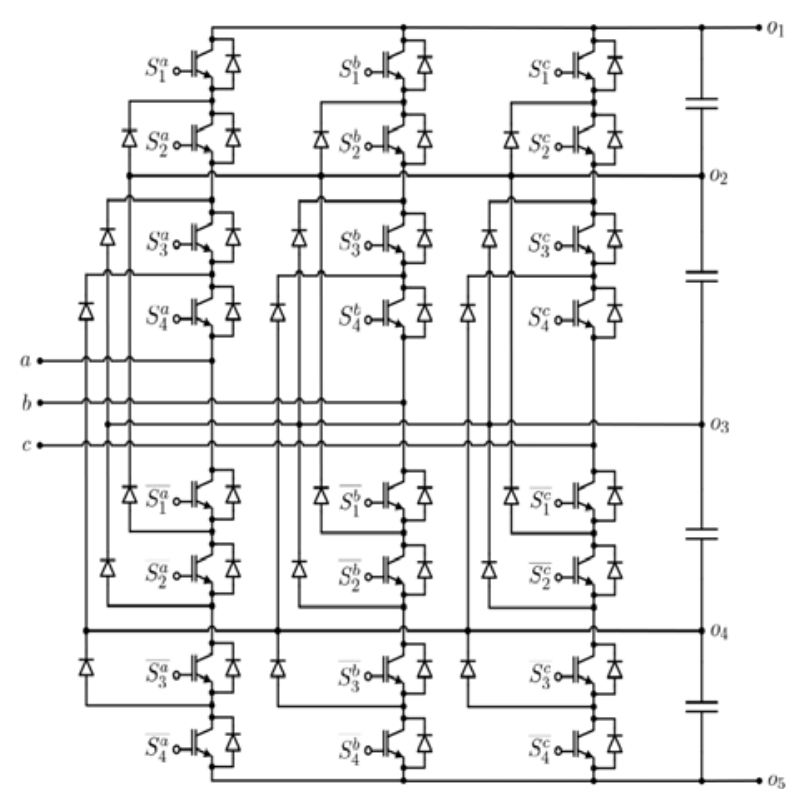

Figura 1: Circuito de un convertidor de cinco niveles de diodos fijos.

El índice de este artículo es el siguiente. En el apartado 2, se hará una descripción del esquema del circuito, se mostrarán las ecuaciones del sistema así como las ecuaciones del controlador. Se mostrarán resultados de simulación del rectificador en el apartado 3. En el apartado 4, se hará una breve descripción de cada uno de los componentes del convertidor y se establecerán las conclusiones en el apartado 5.

\section{ESQUEMA Y MODELO}

En la figura 2 se muestra el convertidor de diodos fijos de cinco niveles funcionando en modo rectificador, que es la configuración que se ha considerado en este trabajo.

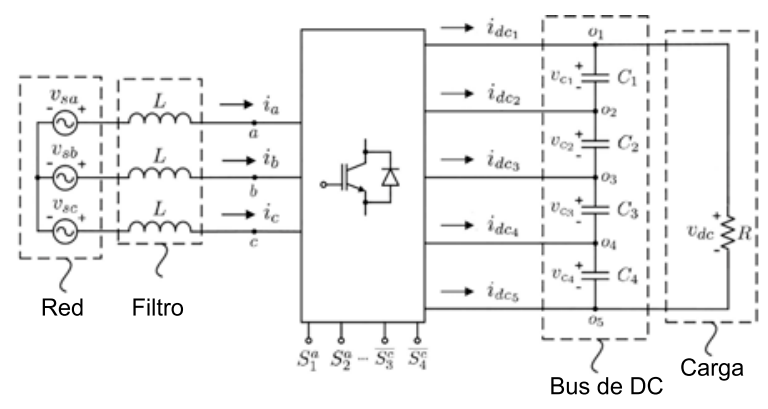

Figura 2: Esquema de un convertidor de cinco niveles de diodos fijos en modo rectificador.

Como puede observarse, el convertidor está conectado a la red a través de un filtro donde las inductancias se consideran de igual valor $L$. $v_{s a}, v_{s b}, v_{s c}, i_{a}, i_{b}$ e $i_{c}$ representan las tensiones $\mathrm{y}$ las corrientes de fase respectivamente.

El bus de continua está compuesto por los condensadores $C_{1}, C_{2}, C_{3}$ y $C_{4}$, todos del mismo valor $C$. Sus tensiones respectivas están representadas por $v_{c 1}, v_{c 2}, v_{c 3}$ y $v_{c 4}$. La suma de estas tensiones representan la tensión total del bus de continua $v_{d c}$. Este lado se encuentra conectado a una carga puramente resistiva $R$.

\subsection{Modelo}

El modelo dinámico del convertidor de cinco niveles fue presentado en [5]. De esta forma, la dinámica de las corrientes de fase, expresadas en coordenadas $\alpha \beta \gamma$, y las diferencias de tensiones en los condensadores se pueden expresar como,

$$
\begin{gathered}
L \frac{d i_{\alpha}}{d t}=v_{\alpha}-u_{1} \frac{v_{d c}}{4}-\left(3 u_{3}-u_{5}+2 u_{7}\right) \frac{v_{d_{1}}}{4} \\
+\left(u_{3}-3 u_{5}+2 u_{7}\right) \frac{v_{d_{2}}}{4}+\left(u_{3}-u_{5}+2 u_{7}\right) \frac{v_{d_{3}}}{2} \\
L \frac{d i_{\beta}}{d t}=v_{\beta}-u_{2} \frac{v_{d c}}{4}-\left(3 u_{4}-u_{6}+2 u_{8}\right) \frac{v_{d_{1}}}{4} \\
+\left(u_{4}-3 u_{6}+2 u_{8}\right) \frac{v_{d_{2}}}{4}+\left(u_{3}-u_{5}+2 u_{8}\right) \frac{v_{d_{3}}}{2} \\
C \frac{d v_{d_{1}}}{d t}=u_{3} i_{\alpha}+u_{4} i_{\beta} \\
C \frac{d v_{d_{2}}}{d t}=u_{5} i_{\alpha}+u_{6} i_{\beta} \\
C \frac{d v_{d_{3}}}{d t}=-u_{7} i_{\alpha}-u_{8} i_{\beta}
\end{gathered}
$$

Donde $u_{1}$ a $u_{8}$ son las señales de control, y las diferencias de tensiones están definidas de la forma:

$$
\begin{aligned}
& v_{d_{1}}=v_{c_{1}}-v_{c_{4}} \\
& v_{d_{2}}=v_{c_{2}}-v_{c_{3}} \\
& v_{d_{3}}=v_{c_{3}}-v_{c_{4}}
\end{aligned}
$$

Utilizando la estrategia de control descrita en [6], se fijan como objetivos de control del convertidor,

- Alcanzar la referencia de tensión en el bus de continua.

- Conseguir que se establezca un equilibrio o balance de tensiones en los condensadores, de forma que las ecuaciones (6)-(8) sean iguales a cero.

- Obtener una señal de intensidades $i_{a}, i_{b}$ e $i_{c}$ lo más senoidal posible, lo que se traduce en un valor de THD bajo. 
En dicha estrategia, se definen las señales de control anteriormente mencionadas como,

$$
\begin{gathered}
u_{1}=\frac{4 k_{p}}{v_{d c}}\left(i_{\alpha}^{r}-i_{\alpha}\right)+\frac{4 k_{i}}{v_{d c}} \int_{0}^{t}\left(i_{\alpha}^{r}-i_{\alpha}\right) d \tau+v_{\alpha} \\
u_{2}=\frac{4 k_{p}}{v_{d c}}\left(i_{\beta}^{r}-i_{\beta}\right)+\frac{4 k_{i}}{v_{d c}} \int_{0}^{t}\left(i_{\beta}^{r}-i_{\beta}\right) d \tau+v_{\beta} \\
u_{3}=-k_{1} i_{\alpha}\left(v_{d_{1}}^{r}-v_{d_{1}}\right) \\
u_{4}=-k_{1} i_{\beta}\left(v_{d_{1}}^{r}-v_{d_{1}}\right) \\
u_{5}=-k_{2} i_{\alpha}\left(v_{d_{2}}^{r}-v_{d_{2}}\right) \\
u_{6}=-k_{2} i_{\beta}\left(v_{d_{2}}^{r}-v_{d_{2}}\right) \\
u_{7}=-k_{3} i_{\alpha}\left(v_{d_{3}}^{r}-v_{d_{3}}\right) \\
u_{8}=-k_{3} i_{\beta}\left(v_{d_{3}}^{r}-v_{d_{3}}\right)
\end{gathered}
$$

donde $k_{p}, k_{i}, k_{1}, k_{2}$ y $k_{3}$ son constantes positivas a seleccionar, el superíndice $r$ indica que son señales de referencia constantes o a calcular como,

$$
\begin{aligned}
& i_{\alpha}^{r}=\frac{1}{v_{\alpha}^{2}+v_{\beta}^{2}}\left(v_{\alpha} p^{r}-v_{\beta} q^{r}\right) \\
& i_{\beta}^{r}=\frac{1}{v_{\alpha}^{2}+v_{\beta}^{2}}\left(v_{\beta} p^{r}+v_{\alpha} q^{r}\right)
\end{aligned}
$$

para una potencia reactiva de referencia igual a cero y una potencia activa de referencia calculada mediante un PI clásico de la forma,

$$
p^{r}=k_{p p}\left(v_{d c}^{r}-v_{d c}\right)+k_{i p} \int_{0}^{t}\left(v_{d c}^{r}-v_{d c}\right) d \tau
$$

\section{SIMULACIÓN}

En este apartado se muestran los resultados de simulación del convertidor que se ha diseñado. A partir de estas simulaciones se han podido calcular o comprobar la validez de alguno de los parámetros utilizados en el diseño del convertidor. Las simulaciones se han realizado con el programa de cálculo matemático y de simulación Matlab Simulink.

Para estas simulaciones se han utilizado los parámetros de la tabla 1 , donde se puede observar, a parte de los parámetros del circuito y de los controladores, que se ha realizado un cambio en la referencia de la tensión de salida del bus de continua en el instante $\mathrm{t}=0.5 \mathrm{~s}$, pasando de $v_{d c}^{r}=800 \mathrm{~V}$ a $v_{d c}^{r}=900 \mathrm{~V}$.

Teniendo en cuenta que se trabaja con un sistema no lineal, los parámetros del controlador se han sintonizado mediante ensayo y error.

Cuadro 1: Parámetros de simulación

\begin{tabular}{ll}
\hline Parámetro & Valor \\
\hline Carga resistiva $(R)$ & $60 \Omega$ \\
Tensión deseada en continua $\left(v_{d c}^{r}\right)$ & $800-900 \mathrm{~V}$ \\
Potencia reactiva deseada $\left(q^{r}\right)$ & $0 \mathrm{VAr}$ \\
Frecuencia de muestreo $\left(f_{s}\right)$ & $5 \mathrm{KHz}$ \\
Frecuencia de conmutación $\left(f_{s w}\right)$ & $5 \mathrm{KHz}$ \\
Ganancia proporcional corriente $\left(k_{p}\right)$ & 10 \\
Ganancia integral corriente $\left(k_{i}\right)$ & 0.1 \\
Ganancia proporcional potencia $\left(k_{p p}\right)$ & 0.1 \\
Ganancia integral potencia $\left(k_{i p}\right)$ & 2.5 \\
Ctes balance de tensiones $\left(k_{1,2,3}\right)$ & $5 \cdot 10^{-4}$ \\
Ctes de transformación $\left(k_{\gamma o 1}, k_{\gamma o 5}\right)$ & 0.7 \\
Ctes de transformación $\left(k_{\gamma o 2}, k_{\gamma o 4}\right)$ & 0.1 \\
\hline
\end{tabular}

Utilizando estos parámetros, se han obtenido los siguientes resultados.

En la figura 3 se representa la tensión total del bus de continua, obtenida como la suma de las tensiones de los condensadores. En esta figura se puede observar como la tensión de salida alcanza fielmente la referencia que se especifica en cada instante.

En las figuras 4, 5 y 6 se pueden observar las diferencias de tensiones $v_{d_{1}}, v_{d_{2}}$ y $v_{d_{3}}$ definidas en (6)-(8). Como se puede observar, estas tensiones se encuentran muy cercanas a cero, garantizando un correcto balance de tensiones en el bus de continua.

Por último, en las figuras 7, 8 y 9 , se puede ver la evolución de las corrientes de fase $i_{a}, i_{b}$ e $i_{c}$ frente a sus respectivas intensidades de referencia $i_{a}^{r}, i_{b}^{r} \mathrm{e}$ $i_{c}^{r}$.

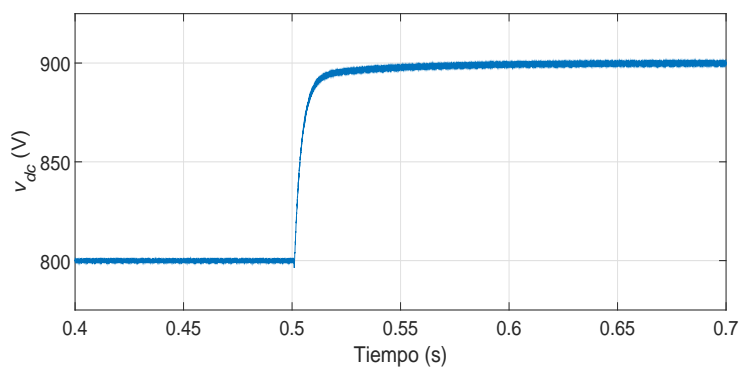

Figura 3: Evolución de la tensión en continua. 


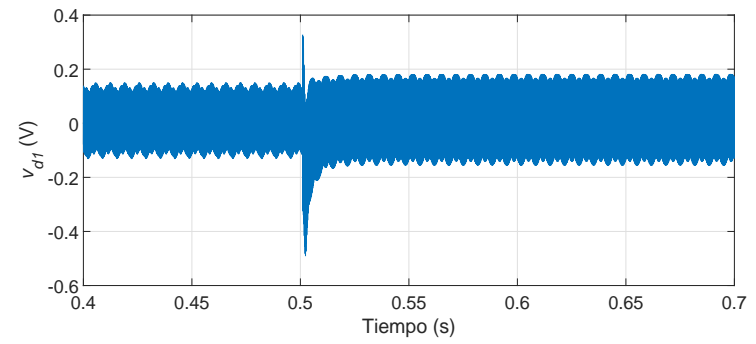

Figura 4: Evolución de la diferencia de tensión en los condensadores $C_{1}$ y $C_{4}$.

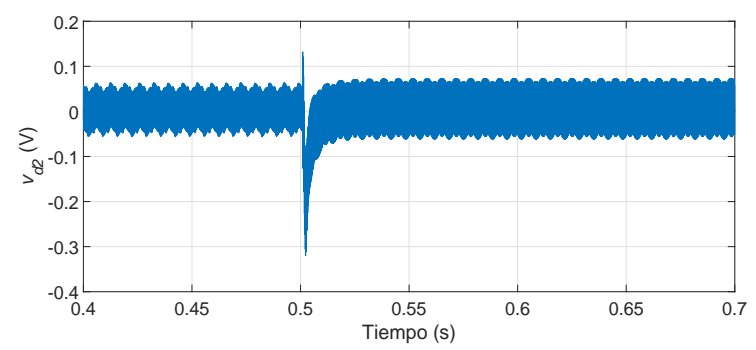

Figura 5: Evolución de la diferencia de tensión en los condensadores $C_{2}$ y $C_{3}$.

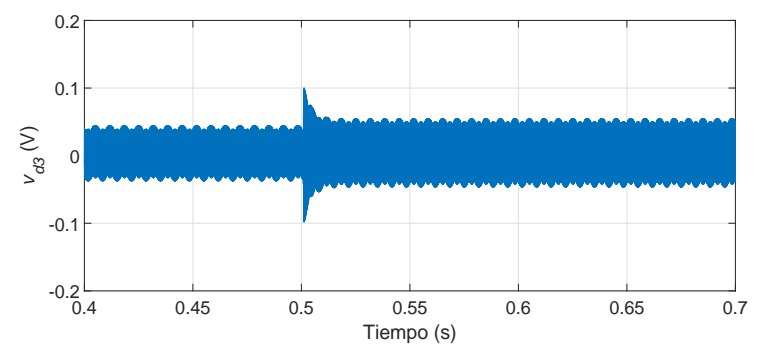

Figura 6: Evolución de la diferencia de tensión en los condensadores $C_{3}$ y $C_{4}$.

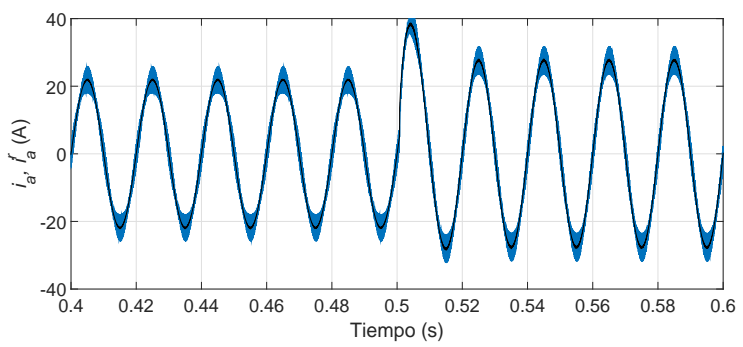

Figura 7: Evolución de $i_{a}$ e $i_{a}^{r}$

\section{DISEÑO Y MONTAJE}

\subsection{Elementos del convertidor}

\subsubsection{Condensadores}

Los condensadores elegidos son el modelo MAL210217332E3 de la marca Vishay. Estos con-

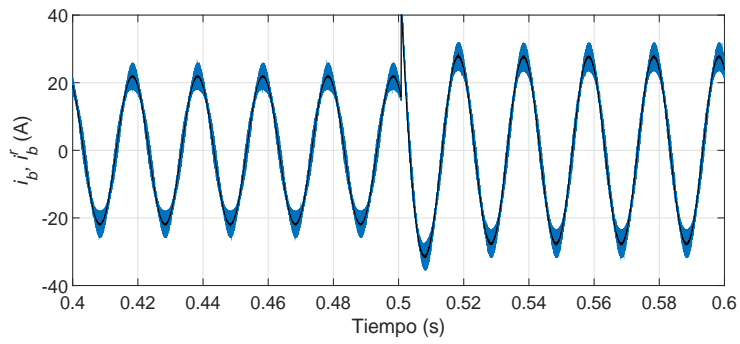

Figura 8: Evolución de $i_{b}$ e $i_{b}^{r}$

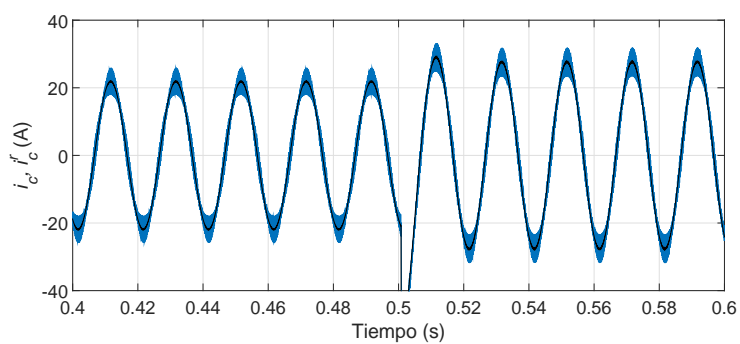

Figura 9: Evolución de $i_{c}$ e $i_{c}^{r}$

densadores electrolíticos tienen una capacidad de $3300 \mu F$ y soportan una tensión de $450 \mathrm{~V}$.

\subsubsection{Filtro}

Como puede observarse en la figura 2, a la entrada del equipo hay dispuestas tres bobinas, una por fase, para el filtrado de la corriente. El valor de su inductancia es de $2 \mathrm{mH}$ cada una. Estas bobinas se encargaron al fabricante de componentes electrónicos Clarkia S.L.

\subsubsection{Sensores}

El convertidor dispone principalmente de dos tipos de sensores necesarios para tareas de control:

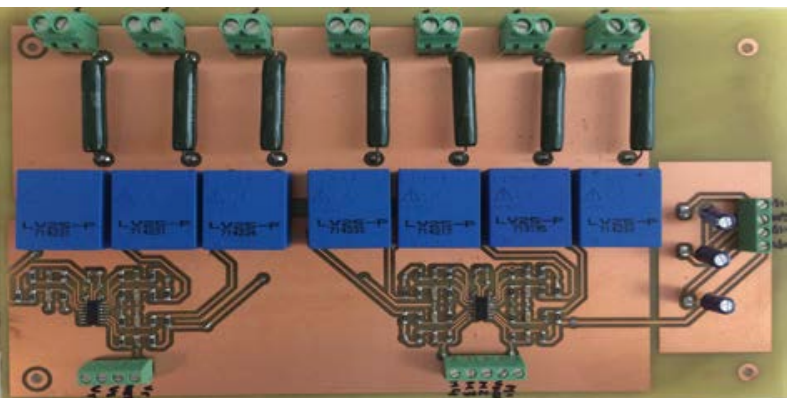

Figura 10: Placa de medida y adaptación de los sensores de tensión.

Sensores de tensión. El sensor utilizado para medida de tensión es de la marca LEM, modelo LV25-800. Estos sensores pueden medir tensiones tanto alterna como continua, por lo que se pue- 
den utilizar para medir las tensiones de red a la entrada y del bus de continua a la salida del convertidor. Al tratarse de sensores de efecto hall, la salida es en corriente por lo que es necesario un circuito de adaptación a una tensión de 0 a $5 \mathrm{~V}$. Este circuito puede observarse en la figura 10.

Sensores de corriente. Para la medida de corriente a la entrada del convertidor se usan sensores de efecto hall de la marca LEM, modelo LA 55-P, debido a su inmunidad frente al ruido magnético. El rango de medida de este sensor es de hasta 70 amperios, puesto que por simulación se han obtenido valores máximos de unos 40 amperios. Estos sensores también necesitan un circuito de adaptación como el mostrado en la figura 11 ya que también generan su salida en corriente.

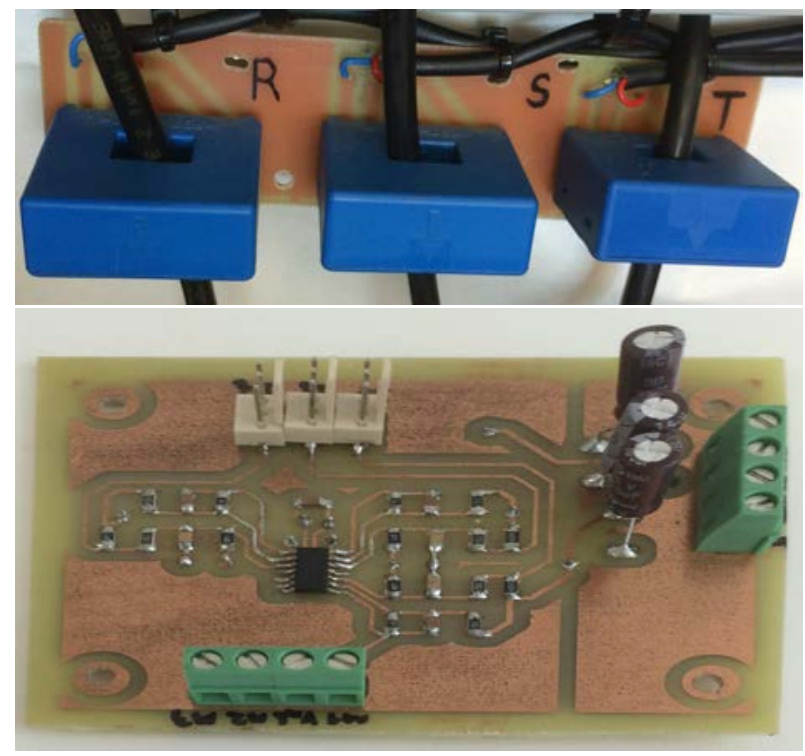

Figura 11: Placa de medida y adaptación de los sensores de corriente.

\subsubsection{Procesador}

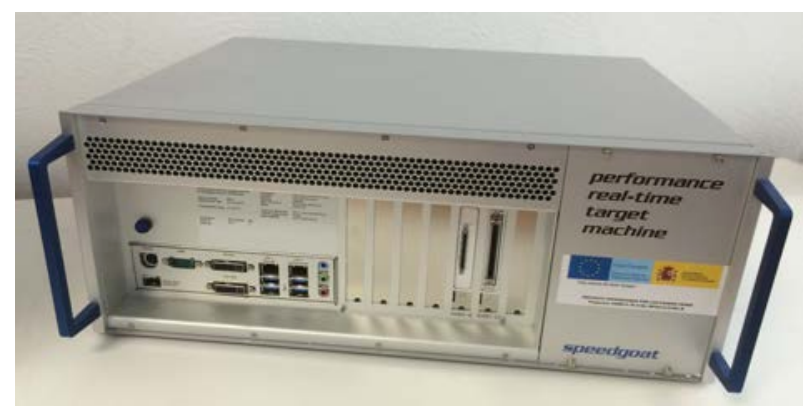

Figura 12: Equipo de control en tiempo real Speedgoat.

Para realizar las tareas de control, se ha utilizado el procesador Speedgoat, el cual es un equipo con el que se pueden realizar experimentos en tiempo real utilizando el programa de simulación Matlab-Simulink. Las características de la Speedgoat, que justifican su uso en este proyecto son varias:

- La posibilidad de realizar el modelo del convertidor y su estrategia de control mediante Simulink.

- La opción de construir y descargar automáticamente una aplicación en tiempo real desde Simulink a la Speedgoat.

- Ajustar los parámetros del controlador, monitorizar el proceso y almacenar datos en tiempo real durante la ejecución de un experimento.

- Trabajar a frecuencias de muestreo altas, lo cual es importante cuando se trabaja con aplicaciones de electrónica de potencia.

La Speedgoat está configurada con un procesador Intel Core i7 de 3.5 Ghz y un módulo FPGA de 75 $\mathrm{MHz}$ que dispone de 128 entradas/salidas. 24 de estas salidas se utilizarán para enviar las señales de control a los actuadores y 6 entradas se utilizarán para enviar posibles señales de error a la Speedgoat.

En la figura 12, se muestra la máquina de tiempo real Speedgoat.

Para transmitir las señales de control desde la Speedgoat, se han utilizado cables de fibra óptica. En los convertidores de potencia, las tensiones de modo común pueden producir el suficiente ruido como para afectar a las señales de control. Por este motivo se ha utilizado fibra óptica, que es inmune a este ruido. De esta forma, la señal de control calculada por la Speedgoat llega a la placa de conversión por el terminal Sub-D en un rango de 0 a 5 voltios y es transformada en una señal de luz que llega a los actuadores a través de los cables de fibra óptica.

En la figura 13 se puede observar esta placa de conversión.

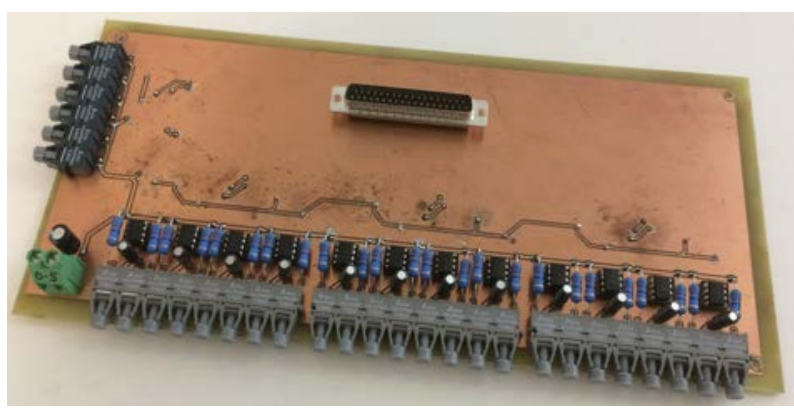

Figura 13: Placa de adaptación de la Speedgoat a fibra óptica. 


\subsubsection{Elementos de conmutación}

Como elemento de conmutación se han utilizado transistores IGBT, que son dispositivos semiconductores que actúan como interruptor controlado por una señal de control. El modelo utilizado en el convertidor es el SKM100GB12V de la marca Semikron. Se han seleccionado estos IGBT que soportan una tensión máxima de 1200 voltios dado que la tensión de trabajo en el bus de continua será de unos 800 voltios. Los IGBT se encuentran encapsulados por pares en unas pastillas como las mostradas en la figura 14.

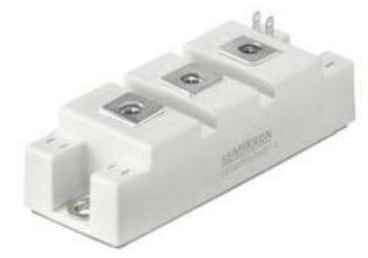

Figura 14: Pastilla de IBGT de Semikron.

Dentro de estas pastillas de IGBT se encuentran también los diodos de conmutación, que son de vital importancia ya que son los encargados de fijar las tensiones de bloque de los interruptores a una fracción de la tensión del bus de continua. Estos diodos son de recuperación rápida y deben soportar la tensión nominal del inversor, por lo que se han escogido unos que soportan hasta 100 amperios.

Una vez enviada la señal de control, calculada por la Speedgoat, a través de fibra óptica, es necesario realizar otra adaptación de la señal a la hora de disparar los IGBT. Esta nueva adaptación se realiza en la placa mostrada en la figura 15. En esta placa, se lee la señal que proviene de la fibra óptica y a través de los drivers se disparan los IGBT. Estos drivers de la marca Semikron y modelo Skyper 32 PRO R, tienen la característica de disparar cada pastilla de IGBT de forma independiente. Además, incorporan protección frente a cortocircuito y apagado suave. Una característica importante, es que no introducen tiempos muertos entre disparos, por lo que para evitar producir un cortocircuito en las conmutaciones será necesario producir estos tiempos muertos desde la Speedgoat.

\subsubsection{Condensadores Snubber}

Son dispositivos condensadores de respuesta en alta frecuencia. Se encuentran en paralelo con los IGBT y sirven para reducir el estrés eléctrico de estos elementos, evitando picos de tensión cuando se realizan las conmutaciones.

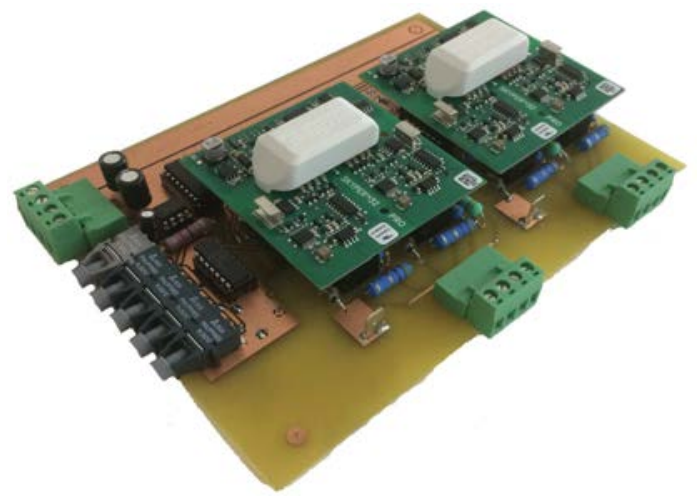

Figura 15: Placa de adaptación de fibra óptica a señal par los IGBT.

\subsubsection{Ventiladores y disipadores}

Tras realizar un estudio térmico sobre el convertidor, se obtuvieron unas temperaturas máximas en las pastillas de IGBT de $147^{\circ} \mathrm{C}$, para un caso con corrientes de prueba de 15 A y tensión de referencia en el bus de continua de 800 V. Para los modelos de pastillas de IGBT y diodos elegidos, la temperatura máxima de trabajo son $150{ }^{\circ} \mathrm{C}$. Con el objetivo de hacer trabajar al equipo con unas condiciones térmicas favorables o en el caso de trabajar con corrientes o tensiones mayores, se decidió incluir disipadores que alojaran, cada uno, tres pastillas de IGBT. Se seleccionó el disipador de calor P3/300B de la marca Semikron con un ventilador axial en uno de sus extremos. Este disipador está especialmente diseñado para el montaje de las pastillas de IGBT en módulos de potencia. Como resultado de la simulación incluyendo estos disipadores y para una temperatura ambiente de $40{ }^{\circ} \mathrm{C}$, se obtuvo una temperatura máxima en el disipador de $95.75^{\circ} \mathrm{C}$, siendo la temperatura final en las pastillas de IGBT de $114.55^{\circ} \mathrm{C}$.

\subsubsection{Contactor}

Como medida de seguridad, se ha colocado un contactor a la entrada del convertidor. De esta forma es posible desconectar la alimentación en caso necesario. Este contactor de la marca Lovato, soporta una potencia de $15 \mathrm{KW}$.

\subsection{Montaje}

En la figura 16, se muestra el estado actual de montaje del convertidor. En esta figura se puede observar que aún falta por conectar todo el cableado, estando las placas electrónicas, sensores, actuadores y demás componentes descritos previamente ya disponibles para su incorporación.

El armario en el que se ha montado el convertidor tiene una altura de 1.8 metros, $60 \mathrm{~cm}$ de ancho y 


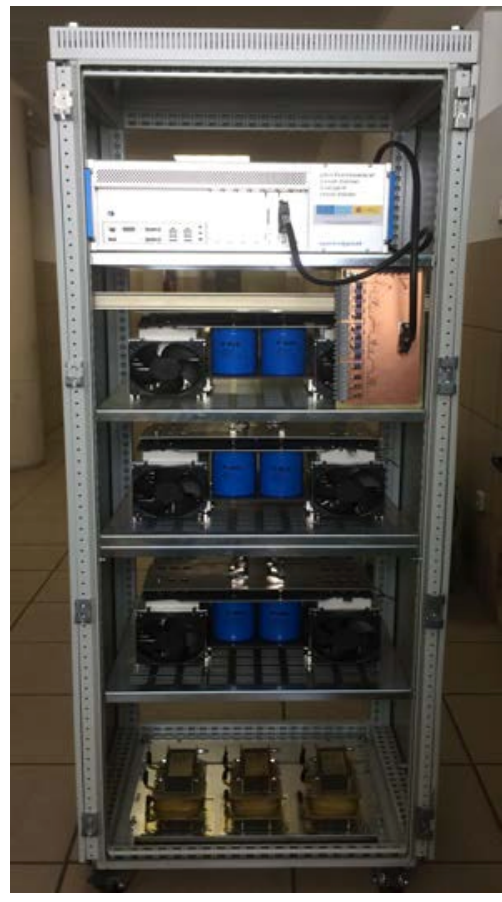

Figura 16: Estado actual del montaje del convertidor.

$60 \mathrm{~cm}$ de fondo.

El convertidor se ha estructurado de forma que cada una de las tres bandejas centrales del armario corresponden a cada una de las fases de la alimentación $a, b$ y $c$ mostradas en las figuras 1 y 2 .

En la figura 17, se puede observar en detalle la construcción de cada bandeja. En ella se puede ver que las conexiones de los condensadores y los IGBT se han establecido, mediante placas de aluminio, en tres planos distintos separados por una capa aislante.

En esta figura se puede ver también la disposición de diferentes elementos descritos en apartados anteriores.

Las bobinas del filtro de la alimentación se han colocado en la bandeja inferior junto a los sensores de corriente, mientras la Speedgoat y los sensores de tensión se han colocado en la bandeja superior junto a las fuentes de alimentación de la electrónica.

\section{CONCLUSIONES}

En este artículo se ha presentado el trabajo realizado para diseñar y montar un rectificador de cinco niveles de diodos fijos. Se ha detallado cada una de las partes y componentes que integran el convertidor y se han realizado simulaciones utilizando las características de éstos.

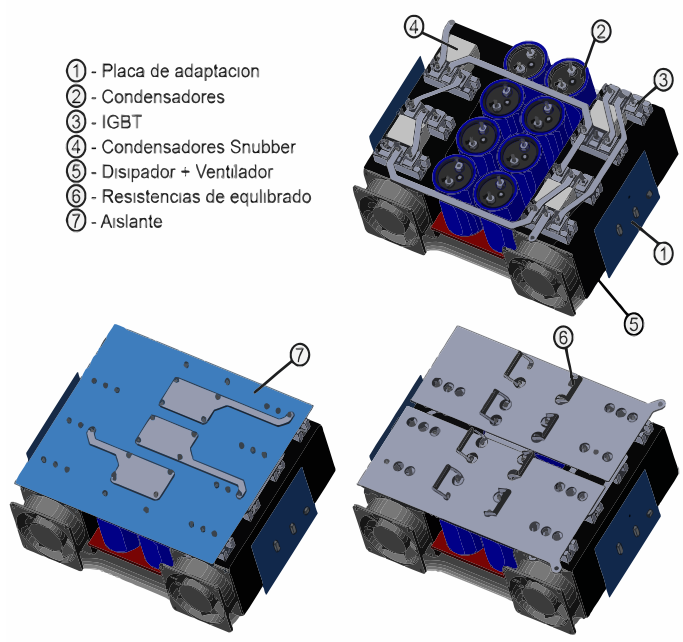

Figura 17: Detalle bandeja de componentes.

Las simulaciones han permitido mostrar los límites físicos de diferentes componentes utilizados en el diseño del convertidor, y a la vez han servido para tener una estimación de la potencia que se podrá obtener del mismo.

Dado que el convertidor se encuentra aún en proceso de montaje, no se ha podido validar la exactitud de las simulaciones respecto al sistema real, tarea que se realizará en un futuro.

\section{Agradecimientos}

Este trabajo se ha realizado bajo la financiación del proyecto MINECO-FEDER DPI2013-41891$\mathrm{R}$.

\section{Referencias}

[1] Lai, J. S. y Peng, F. Z. (1995). Multilevel converters-a new breed of power converters. IAS '95. Conference Record of the 1995 IEEE Industry Applications Conference Thirtieth IAS Annual Meeting, 3:2348-2356. IEEE.

[2] Kouro, S., Malinowski, M., Gopakumar, K., Pou, J., Franquelo, L. G., Rodriguez, J., Perez, M. A. y Leon, J. I. (2010). Recent Advances and Industrial Applications of Multilevel Converters. IEEE Transactions on Industrial Electronics, 57(8):2553-2580.

[3] Rodriguez, J., Bernet, S., Wu, B., Pontt, J. O. y Kouro, S. (2007). Multilevel VoltageSource-Converter Topologies for Industrial Medium-Voltage Drives. IEEE Transactions on Industrial Electronics, 54(6):2930- 2945.

[4] Rodriguez, J., Lai, J. S. y Peng, F. Z. (2002). Multilevel Inverters: A Survey 
of Topologies, Controls, and Applications. IEEE Transactions on Industrial Electronics, 49(4):724-738.

[5] Umbría, F., Gordillo F. y Salas, F. (2016). A controller for practical stability of capacitor voltages in a five-level diodeclamped converter, European Journal of Control, 28:56-68, ISSN 0947-3580, http://dx.doi.org/10.1016/j.ejcon.2015.10.004.

[6] Umbría, F., Gordillo F. y Salas, F. (2016). Modelling and Decoupling Controll of a GridConnected Five-Level Diode-Clamped Converter, Electric Power Components and Systems, Pendiente de publicar.

[7] Zargari, N. y Rizzo, S. (2004). Medium Voltage Drives in Industrial Applications. Proc. Tech. Seminar, IEEE Toronto Section. 